Volume 1 Issue 1

June 2016

\title{
Clinical Update: Improving Cardiac Arrest Care in Ireland
}

David Hennelly MSc

Clinical Development Manager, National Ambulance Service, Ireland; david.hennelly@hse.ie

\section{Recommended Citation}

Hennelly D. Improving Cardiac Arrest Care in Ireland. Irish Journal of Paramedicine. 2016 Jun; 1(1)

This is an Open Access article distributed under the terms of the Creative Commons Attribution-Non-Commercial-ShareAlike 4.0 International (http://creativecommons.org/licenses/by-nc-sa/4.0/), which permits use, distribution, and reproduction in any medium, provided the original work and any attributes thereof are properly cited, are distributed under the same licence, and that the work is not used for commercial purposes.

Follow the Irish Journal of Paramedicine online at www.irishparamedicine.com, on Twitter (@irishjparamed) and on Facebook. 
CLINICAL UPDATE

\title{
Improving Cardiac Arrest Care in Ireland
}

\author{
David Hennelly MSc ${ }^{1}$ \\ 1. Medical Directorate, National Ambulance Service, Ireland.
}

Received: 14 February 2016

Revised: n/a

Accepted: 19 March 2016

Published: 01 June 2016

Correspondence: David Hennelly, National Ambulance Service, Dooradoyle, Limerick, Ireland. Email: david.hennelly@hse.ie

\section{Abstract}

Over the past two years the National Ambulance service has engaged with both the country's leading resuscitation experts and our international counterparts in a collaborative project focused on developing and expanding the National Ambulance Services response to out of hospital cardiac arrest. The One Life Project not only represents our commitment to improve standards of care it also represents our commitment to measure and publicly report on clinical outcomes of patients.

\section{Keywords: resuscitation; cardiac arrest; OHCA; CPR; Ireland; National Ambulance Service}

\section{Introduction}

The National Ambulance Service (NAS) serves a population of almost 4.6 million people in the Republic of Ireland, the service responds to over 300,000 ambulance calls each year. The NAS employs over 1,600 staff across 100 locations and has a fleet of approximately 500 vehicles. Emergency Medical Services attempt resuscitation on just under 2000 Out of Hospital Cardiac Arrest (OHCA) cases each year.

Over the past two years the National Ambulance service has engaged with both the country's leading resuscitation experts and our international counterparts in a collaborative project focused on developing and expanding the National Ambulance Services response to out of hospital cardiac arrest. The One Life Project not only represents our commitment to improve standards of care it also represents our commitment to measure and publicly report on clinical outcomes of patients.

\section{Strategy}

The implementation strategy is broken into four strategic pillars (Figure 1).

\section{NAS community interaction and public education}

The National Ambulance Service continues to support and grow its Community First Responder (CFR) network and currently has in excess of 140 linked CFR Schemes. The role of the One Life Project has been to support the growth of CFR groups linked to the NAS and to seek out complementary methods of community response, such as; increasing Public Access Defibrillator (PAD) registration, activation of off duty NAS practitioners, activation of other healthcare professionals and responders and exploring the evolving arena of social media responders.

We have had an excellent response from our medical colleagues in General Practice. In conjunction with University College Dublin MERIT program we have now almost 100 General Practitioners across the country linked to the NAS to receive text alert to OHCA cases in close proximity to their workplace.

This area also focuses on the interaction and education of the public, from growing support for schools CPR programs in association with the Irish Heart Foundation to a significant increase in our services promotion of the chain of survival via our social media outlets on Twitter and Facebook

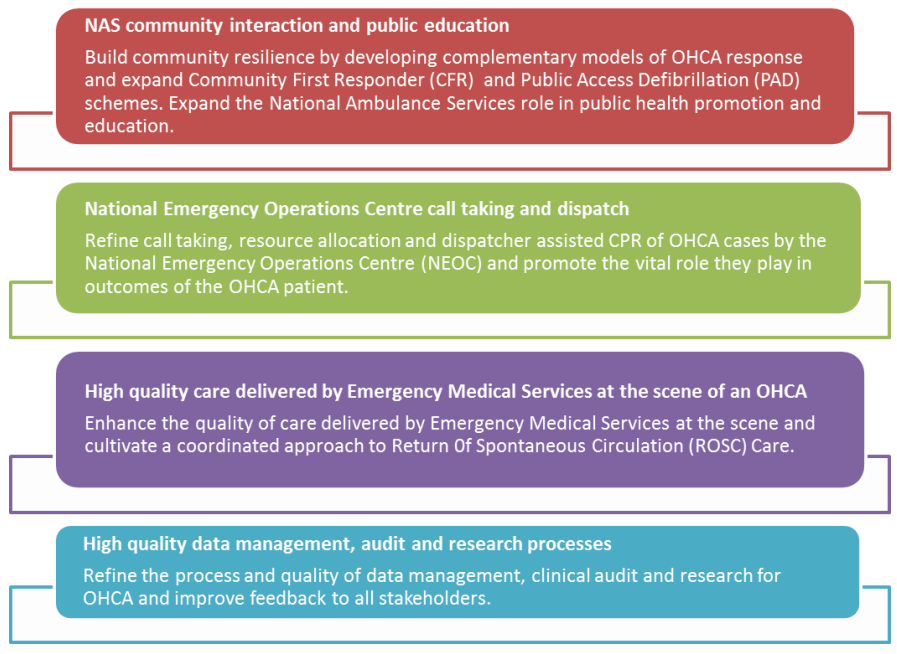

Figure 1. NAS One Life Implementation Strategy

\section{National Emergency Operations Centre call taking and dispatch}

When an emergency ambulance call is received, the Call Taker within the National Emergency Operations Centre (NEOC) uses a medical priority dispatch system (MPDS) to triage the call to determine the clinical priority and the appropriate response required. A coded response system is used, based on international best practice.

Life threatening calls, such as a cardiac or respiratory arrest take precedence over all other calls. The closest available resource is immediately dispatched, such as a NAS Emergency Ambulance, NAS Rapid Response Vehicle, NAS Intermediate Care Vehicles, or a local Community First Responder (CFR) group or doctor. For a suspected cardiac arrest the NAS Call Taker will instruct the person making the call, how to perform CPR and how to use an automated external defibrillator (AED) where available to the person.

The NEOC is continually striving to enhance the role they play in each OHCA, focusing on key areas of impact such as, early recognition of the cardiac arrest patient, increasing bystander $\mathrm{CPR}$ rates and rapid activation of multiple responders. This has been achieved through ongoing 
education and performance review and investment in the newest software support MPDS v13.0 which incorporates a Fast Track to "hands-on-chest" for suspected OHCA cases.

This continued focus on improving outcomes from cardiac arrest has seen a continued rise in bystander CPR rates from $60 \%$ in 2012 to $71 \%$ in 2014 .

\section{High quality care delivered by Emergency Medical Services at the scene of an OHCA}

The One Life Project has developed a comprehensive position paper on the optimal scene management of an out of hospital cardiac arrest which will be released early in 2016 in line with clinical practice guideline updates on the 2015 ILCOR guidelines. The document and supporting material focuses on the best evidence in resuscitation care outlining the principles of high performance resuscitation and human factors management, the objective of which is to improve the quality and dignity of care delivered by NAS practitioners at the scene.

This process includes a renewed emphasis on resuscitation training in 2016 where NAS Education and Competency Assurance Teams (ECAT) will be introducing the principles high-performance resuscitation into each OHCA. This includes a well-planned, well-rehearsed and often choreographed approach to caring for the victim of sudden cardiac arrest with an increased focus on using a team approach; human factors training and the use of pre determine roles and checklists during resuscitation at the scene.

This quality improvement process is running concurrently with a significant investment by the NAS in a single monitor/defibrillator solution for the entire service (Physio Control LIFEPAK ${ }^{\circledR} 15$ ) and an increase in the number of mechanical compression devices (LUCAS2 $\AA$ ). This investment is providing our service with the opportunity to standardise the equipment used during each resuscitation and a greater opportunity to capture data at the scene and analyse our performance via CODE-STAT ${ }^{\mathrm{TM}} 9.0$ data review software.

The One Life Project has also introduced post return of spontaneous circulation (ROSC) checklists, optimising the care of the post ROSC patient. This incorporates the principle outlined in the 2015 ILCOR guidelines, including optimising oxygenation, ventilation, haemodynamic and neurologic status and targeting those STEMI patients who require direct admission to primary percutaneous coronary intervention.

\section{High quality data management, audit and research processes}

Ireland is one of only three European countries with a single national OHCA registry. Since its inception in 2007, the Out-of-Hospital Cardiac Arrest Registry (OHCAR) has grown from a regional register in the North West to a nationwide register in 2012, incorporating OHCA data from statutory and voluntary services across Ireland. One of the early innovations of the One Life Project was the identification of the issues surrounding OHCAR data collection and processing.

Until this point there had been no single approach to the collation of OHCAR data from each region within the NAS. The One Life Project has developed and instituted a national process of data collection, regional collation and data processing; with collaboration from OHCAR the NAS can now track each OHCA event from inception to conclusion and has significantly reduced the data processing required and allowed greater emphasis on Validation of the data and feedback to the appropriate stakeholders.

In November 2015 the NAS Medical Directorate introduced quarterly OHCA infographics to highlight the important work NAS practitioners are carrying out in each of their own areas. The One Life Project will continue to work with OHCAR to maximise the data collection and processing in line with future technological advances.

\section{Summary}

The benefits to patients and their families remain the overarching focus of the One Life Project. Our service's goal is to treat effectively as many people in cardiac arrest as possible so that they are neurologically intact and ready to resume their role within their family and society. Since the introduction of this quality improvement initiative we have seen a steady rise in the percentage ROSC at ED rates, from $24 \%$ in 2014 to $42 \%$ at the end of 2015 , this is down to the consistent professional effort by our front line call takers, dispatchers and practitioners.

By working together, the implementation of this project will improve patient outcomes for each cardiac arrest call and dramatically increase our chances of saving that 'One Life'.

Source of support/funding: National Ambulance Service, Ireland.

Conflict of interest: None declared.

Provenance and review: Not commissioned, peerreviewed.

Editor's Note: This article originally appeared in Resuscitation Today, 3(1). Reprinted with permission.

How to cite this article: Hennelly D. Improving Cardiac Arrest Care in Ireland. Irish Journal of Paramedicine 2016; 1 (1).

This is an Open Access article distributed under the terms of the Creative Commons Attribution-Non-Commercial-ShareAlike 4.0 International (http:// creativecommons.org/licenses/by-nc-sa/4.0/), which permits use, distribution, and reproduction in any medium, provided the original work and any attributes thereof are properly cited, are distributed under the same licence, and that the work is not used for commercial purposes. Content copyright remains with the authors, who grant the IJP a licence to reuse and distribute. 